\title{
APPROXIMATION OF OPTIMAL REINSURANCE AND DIVIDEND PAY-OUT POLICIES
}

\author{
NICOLE BÄUERLE \\ Faculty of Mathematics and Economics, University of Ulm, D-89069 Ulm, Germany
}

We consider the stochastic process of the liquid assets of an insurance company assuming that the management can control this process in two ways: first, the risk exposure can be reduced by affecting reinsurance, but this decreases the premium income. Second, a dividend has to be paid out to the shareholders. The aim is to maximize the expected discounted dividend pay-out until the time of bankruptcy. The classical approach is to model the liquid assets or risk reserve process of the company as a piecewise deterministic Markov process. However, within this setting the control problem is very hard. Recently several papers have modeled this problem as a controlled diffusion, presuming that the policy obtained is in some sense good for the piecewise deterministic problem as well. We will clarify this statement in our paper. More precisely, we will first show that the value function of the controlled diffusion provides an asymptotic upper bound for the value functions of the piecewise deterministic problems under diffusion scaling. Finally it will be shown that the upper bound is achieved in the limit under the optimal feedback control of the diffusion problem. This property is called asymptotic optimality.

KEY WorDs: dividend pay-out, proportional reinsurance, piecewise deterministic process, diffusion process, asymptotic optimality, Hamilton-Jacobi-Bellman equation

\footnotetext{
${ }^{0}$ The author would like to thank Paul Embrechts for a fruitful discussion and two referees for valuable comments.
} 


\section{Introduction}

A classical problem in actuarial mathematics deals with the question of optimal dividend pay-out of an insurance company. Earlier papers on this subject include among others de Finetti (1957), Borch $(1967,1969)$, Bühlmann (1970) and Gerber (1972, 1979). One main motivation for this problem is that according to a theorem of Miller and Modigliani (1961) the value of a company is equal to the present value of the net distributions to shareholders over an infinite horizon. Sethi et al. (1991) and Sethi (1996) have shown the validity of this theorem in a stochastic setting. The standard framework for this problem is to model the liquid assets or risk reserve process of the company as a piecewise deterministic Markov process. Within this setting the control problem is very hard. An explicit solution cannot be found. Thus Dassios and Embrechts (1989), for example, determine the optimal dividend policy among all threshold policies, and the main result of Schäl (1998) proves the existence of an optimal feedback control and characterizes it as a maximizer of a Hamilton-Jacobi-Bellman equation.

Another interesting question concerns the optimal risk exposure of the company. Here it is assumed that the management of an insurance company can determine the amount of reinsurance. Again the solution in a piecewise deterministic framework is considered, but this is hard (see e.g. Schäl (1998), Hipp and Vogt (2001), Schmidli (2001, 2002)). Schäl (1998) treats these two problems simultaneously.

Recently there has been a renewed significant interest in the problem of optimal dividend pay-out and risk management of (insurance) companies, see e.g. Asmussen and Taksar (1997), Højgaard and Taksar (1998, 1999, 2001), Asmussen et al. (2000). The reason is that motivated by papers of Radner and Shepp (1996) and Browne (1995, 1997) the authors now model the risk reserve process as a controlled diffusion. This approach often allows one to compute an explicit optimal control and a smooth value function. The optimal control is then supposed to work well for the original controlled piecewise deterministic process. However, this statement is not trivial, and we will show in this paper in which sense and under what assumptions it is true.

Let us now start with the mathematical formulation of the (original) piecewise deterministic Markov model. As in the classical risk model of Cramér and Lundberg (see e.g. Grandell (1991)) we suppose that the insurance company has a fixed, deterministic premium income rate $c>0$ and claims arrive according to a Poisson process $\left(N_{t}\right)$ with intensity $\lambda>0$. The size of the $n$-th claim is given by the random variable $Y_{n} \geq 0$. We suppose that the $\left\{Y_{n}\right\}$ are independent and identically distributed with distribution function $F$ and that $m:=\int_{0}^{\infty} x d F(x)$ and $s^{2}:=\int_{0}^{\infty}(x-m)^{2} d F(x)$ are finite. All random elements should be defined on a common probability space $(\Omega, \mathcal{F}, P)$. By $\left\{\mathcal{F}_{t}\right\}_{t \geq 0}$ we denote the natural filtration which is induced by $\left(N_{t}\right)$ and $\left(Y_{1}, \ldots, Y_{N_{t}}\right)$. The compound Poisson process is denoted by $S_{t}:=\sum_{i=1}^{N_{t}} Y_{i}$. As in Dassios and Embrechts (1989) (cf. also Embrechts and Schmidli (1994), Højgaard and Taksar (2001), Schäl (2002) ) we suppose that the company earns interest on the (invested) risk reserve. The rate of interest for 
invested money is supposed to be $\beta_{0}$ and we define

$$
\rho(x)= \begin{cases}0, & x<0 \\ \beta_{0} x, & x \geq 0\end{cases}
$$

$\rho(x)$ represents the interest which is gained for the invested capital $x$ in the case $x \geq 0$. If the risk reserve $x$ is negative $(x<0), \rho(x)$ tells us that money can be borrowed at no cost. The stochastic differential equation (SDE) for the uncontrolled risk reserve is

$$
d R_{t}^{1}=\left(c+\rho\left(R_{t}^{1}\right)\right) d t-d S_{t}
$$

where $R_{0}^{1}=x$ is the initial risk reserve. The superscript ${ }^{1}$ will become clear at the end of this introduction.

Now suppose we are able to control the risk reserve process in the following way. A stochastic control process $\left(\pi_{t}^{1}\right):=\left(\alpha_{t}^{1}, \delta_{t}^{1}\right)$ consists of two parts: $\delta_{t}^{1} \in[0, \Delta]$ is the dividend rate which is paid at time $t$ (the maximal rate is restricted to $\Delta$ ) and $\alpha_{t}^{1} \in[0,1]$ is the deductible in percent of a proportional reinsurance. Thus, if at time $T_{i}$ a claim $Y_{i}$ arrives, the company only has to cover the amount $\alpha_{T_{i}}^{1} Y_{i}$, because $\left(1-\alpha_{T_{i}}^{1}\right) Y_{i}$ is the reinsured part. The premium which has to be paid for this reinsurance at time $t$ is $c\left(1-\alpha_{t}^{1}\right)$. The dynamics for the controlled risk reserve $R_{t}^{\pi^{1}}$ are then given by

$$
d R_{t}^{\pi^{1}}=\left(c \alpha_{t}^{1}+\rho\left(R_{t}^{\pi^{1}}\right)-\delta_{t}^{1}\right) d t-\alpha_{t}^{1} d S_{t},
$$

with $R_{0}^{\pi^{1}}=x$. Here $\pi^{1}=\left(\alpha^{1}, \delta^{1}\right)$ is called policy if it is adapted w.r.t. the filtration $\left\{\mathcal{F}_{t-}\right\}_{t \geq 0}$ and $\left(\alpha_{t}^{1}, \delta_{t}^{1}\right) \in[0,1] \times[0, \Delta]$ for all $t$. Thus, of course, the deductible has to be chosen before we know whether or not a claim arrives. For a given policy $\pi^{1}$, the expected discounted dividend pay-out with initial risk reserve $x$ is given by

$$
V_{\pi^{1}}(x)=E\left[\int_{0}^{\tau_{\pi^{1}}} e^{-\beta t} \delta_{t}^{1} d t\right]
$$

where $\tau_{\pi^{1}}=\tau\left(R_{t}^{\pi^{1}}\right):=\inf \left\{t \geq 0 \mid R_{t}^{\pi^{1}}<0\right\}$ is the time of ruin and $\beta>0$ is the discount rate. The dividend can only be paid out until ruin occurs, which is the case if the risk reserve falls below 0 . The objective is to maximize the expected discounted dividend pay-out

$$
V^{1}(x)=\sup _{\pi^{1}} V_{\pi^{1}}^{1}(x)
$$

over all policies $\pi^{1} . \pi^{1 *}$ is called optimal if $V_{\pi^{1 *}}^{1}(x)=V^{1}(x)$ for all $x$. This original problem is a so-called piecewise deterministic Markov decision process and can be reduced to a discrete-time optimization problem. For details concerning the solution theory of these problems see e.g. Davis (1993).

Next we define the same problem in a diffusion setting. Suppose that $\left\{\mathcal{G}_{t}\right\}_{t \geq 0}$ is a second filtration, $\mathcal{G}_{t} \subset \mathcal{F}$ and $\left(B_{t}\right)$ is a standard Brownian motion with respect to $\left\{\mathcal{G}_{t}\right\}_{t \geq 0}$. We assume that the uncontrolled risk reserve evolves according to

$$
d R_{t}=\left(\mu+\rho\left(R_{t}\right)\right) d t-\sigma d B_{t}
$$


where $\mu=c-\lambda m$ and $\sigma^{2}=\lambda\left(m^{2}+s^{2}\right)$. Note that the definition of $\mu$ is such that the expected drift of the piecewise deterministic risk reserve and the diffusion risk reserve are equal. Here, a policy $\pi$ consists of two $\left\{\mathcal{G}_{t}\right\}_{t \geq 0}$-adapted processes $\left(\alpha_{t}\right)$ and $\left(\delta_{t}\right)$, where $\alpha_{t} \in[0,1]$ gives the deductible at time $t$ and $\delta_{t} \in[0, \Delta]$, the dividend rate at time $t$. The controlled diffusion evolves according to

$$
d R_{t}^{\pi}=\left(\mu \alpha_{t}+\rho\left(R_{t}^{\pi}\right)-\delta_{t}\right) d t-\sigma \alpha_{t} d B_{t}
$$

with $R_{0}^{\pi}=x$. For a given policy $\pi$, the expected discounted dividend pay-out with initial risk reserve $x$ is defined by

$$
W_{\pi}(x)=E\left[\int_{0}^{\tau_{\pi}} e^{-\beta t} \delta_{t} d t\right]
$$

where $\tau_{\pi}=\tau\left(R_{t}^{\pi}\right):=\inf \left\{t \geq 0 \mid R_{t}^{\pi}<0\right\}$ is the time of ruin. As before, the objective is to maximize the expected discounted dividend pay-out

$$
W(x)=\sup _{\pi} W_{\pi}(x)
$$

over all policies $\pi$. Here $\pi^{*}$ is called optimal if $W_{\pi^{*}}(x)=W(x)$ for all $x$. For the theory of controlled diffusion see among others Krylov (1980), Fleming and Rishel (1975) or Fleming and Soner (1993).

It is now well-known that under suitably defined diffusion scaling with parameter $\gamma>0$, the uncontrolled risk reserve processes $\left\{\left(R_{t}^{\gamma}\right)\right\}$ converge weakly to $\left(R_{t}\right)$ (see e.g. Grandell (1977), Harrison (1977)), Schmidli (1994)). Thus, $\left(R_{t}\right)$ can be thought of as an approximation for the original Cramér-Lundberg risk reserve $\left(R_{t}^{1}\right)$. Moreover, as we will see in section 2, the optimization problem can be solved much easier in the diffusion setting. As a result several authors have restricted their analysis to the diffusion setting and proposed that the optimal feedback control obtained there should be a good or reasonable control for the original piecewise deterministic Markov decision process. However, this conclusion is in general dangerous. There are examples from stochastic networks where such a procedure leads to an incredible bad behavior of the policy in the original model. For instance, it has been shown in Maglaras (2000) that the optimal scheduling policy obtained from the first oder (fluid) approximation of a two station Rybko-Stolyar network does not even stabilize the original discrete network, even though a stable policy can be found.

Let us next explain how the diffusion scaling works. The scaling parameter for the risk reserve process is denoted by $\gamma>0$. In the scaled system the intensity with which claims arrive is linearly increased by $\gamma$, i.e. $\lambda_{\gamma}=\lambda \cdot \gamma$. The scaled claim sizes are decreased by $\sqrt{\gamma}$, i.e., it holds for the claim size distribution function $F_{\gamma}(t)=F(\sqrt{\gamma} t)$. The premium income rate is increased by $\sqrt{\gamma}$, i.e. $c_{\gamma}=(\rho+\sqrt{\gamma}) \lambda m$, where $\rho=\frac{c-\lambda m}{\lambda m}$ is the usual safety load. For $\gamma=1$ we obtain our original data (this is the reason for the superscript ${ }^{1}$ ). The economic interpretation of the scaling is that the portfolio or the number of insured risks increases with $\gamma$. Thus, it is quite realistic to assume that $\gamma$ is large in practice, reflecting a large insurance company. A simulation of the uncontrolled 
risk reserve $\left(R_{t}^{\gamma}\right)$ for different parameter $\gamma$ can be seen in figure 1.1. For fixed $\gamma$, the risk reserve process is again a piecewise deterministic Markov process with data $\lambda_{\gamma}, F_{\gamma}$ and $c_{\gamma}$ depending on $\gamma$. Thus, policies $\pi^{\gamma}=\left(\alpha^{\gamma}, \delta^{\gamma}\right)$ for the system with $\gamma$ are defined as before, as well as the optimization problem. Let $\pi^{\gamma}$ be an arbitrary policy. The scaled risk reserve process under policy $\pi^{\gamma}$, denoted by $\left(R_{t}^{\pi^{\gamma}}\right)$, is given by the SDE

$$
d R_{t}^{\pi^{\gamma}}=\left(c_{\gamma} \alpha_{t}^{\gamma}+\rho\left(R_{t}^{\pi^{\gamma}}\right)-\delta_{t}^{\gamma}\right) d t-\frac{1}{\sqrt{\gamma}} \alpha_{t}^{\gamma} d S_{t \gamma}
$$

or, in other terms,

$$
d R_{t}^{\pi^{\gamma}}=\left(\mu \alpha_{t}^{\gamma}+\rho\left(R_{t}^{\pi^{\gamma}}\right)-\delta_{t}^{\gamma}\right) d t-\alpha_{t}^{\gamma} d M_{t}^{\gamma},
$$

where $M_{t}=S_{t}-\lambda m t$ is a martingale and $M_{t}^{\gamma}=\frac{M_{t \gamma}}{\sqrt{\gamma}}$. The scaled expected discounted dividend pay-out under policy $\pi^{\gamma}$ is now denoted by $V_{\pi^{\gamma}}^{\gamma}$, and $V^{\gamma}(x)=\sup _{\pi^{\gamma}} V_{\pi^{\gamma}}^{\gamma}(x)$ is the corresponding value function. In section 3 we will show under some assumptions that $\limsup _{\gamma \rightarrow \infty} V^{\gamma}(x) \leq W(x)$, i.e. the value function of the controlled diffusion provides an asymptotic upper bound. The aim now is to find a sequence of policies $\left\{\pi^{\gamma}\right\}$ such that $\lim _{\gamma \rightarrow \infty} V_{\pi^{\gamma}}^{\gamma}(x)=W(x)$. Of particular interest are so-called feedback rules. A feedback rule $\varphi=(\alpha, \delta)$ gives the current action as a function of the current state. More precisely, a feedback rule $\varphi=(\alpha, \delta)$ is a pair of measurable mappings $\delta: \mathbb{R} \rightarrow[0, \Delta]$ and $\alpha: \mathbb{R} \rightarrow[0,1]$ such that the SDE

$$
d R_{t}^{\varphi, \gamma}=\left(\mu \alpha\left(R_{t}^{\varphi, \gamma}\right)+\rho\left(R_{t}^{\varphi, \gamma}\right)-\delta\left(R_{t}^{\varphi, \gamma}\right)\right) d t-\alpha\left(R_{t}^{\varphi, \gamma}\right) d M_{t}^{\gamma}
$$

has a unique (strong) solution. In our model a feedback rule $\varphi$ for the controlled diffusion is also a feedback rule for the piecewise deterministic Markov process for any $\gamma$. A feedback rule $\varphi$ is called asymptotically optimal if $\lim _{\gamma \rightarrow \infty} V_{\varphi}^{\gamma}(x)=W(x)$.

Our paper is organized as follows. In section 2 we review the existing results concerning the controlled diffusion problem. Section 3 contains our main results. In a first part we summarize several propositions concerning weak convergence of certain processes and of solutions of SDEs. In subsection 3.2 we prove that $W$ is an asymptotic upper bound for $V^{\gamma}$ (Theorem 3.6) and show the asymptotic optimality of the feedback rule $\varphi^{*}$ which solves the controlled diffusion problem (1.6) (Theorem 3.7). Finally, section 4 contains a numerical example and some more words concerning the economic interpretation of our findings.

\section{The Limiting Problem - a Controlled Diffusion. Review of Existing Results}

In this section we will give a short summary of the existing results in the literature which concern the controlled diffusion problem.

The plain dividend pay-out problem (without reinsurance) has been solved by Asmussen and Taksar (1997). The authors showed that a threshold control is optimal. The plain risk exposure problem with proportional reinsurance has been solved by Højgaard 
and Taksar (1998) for the objective of maximizing the expected discounted dividend pay-out. The paper which combines dividend pay-out and risk exposure is Højgaard and Taksar (1999). They consider the diffusion problem introduced in section 1 with $\beta_{0}=0$. Depending on the parameters, the optimal dividend pay-out feedback rule $\delta^{*}$ and the optimal proportional reinsurance $\alpha^{*}$ are given as follows.

If $\Delta<\frac{\mu}{2}+\frac{\beta \sigma^{2}}{\mu}$ then

$$
\delta^{*}(x)= \begin{cases}0, & \text { if } x<m \\ \Delta, & \text { if } x \geq m\end{cases}
$$

with $m=\frac{1}{\beta}(\Delta \nu(1-\nu))$ and $\nu=\frac{\beta}{\frac{\mu^{2}}{2 \sigma^{2}}+\beta}$ and

$$
\alpha^{*}(x)= \begin{cases}\frac{\mu x}{\sigma^{2}(1-\nu)}, & \text { if } x<m \\ \frac{\mu m}{\sigma^{2}(1-\nu)}, & \text { if } x \geq m .\end{cases}
$$

If $\Delta \geq \frac{\mu}{2}+\frac{\beta \sigma^{2}}{\mu}$ then

$$
\delta^{*}(x)= \begin{cases}0, & \text { if } x<m \\ \Delta, & \text { if } x \geq m\end{cases}
$$

with $m=\frac{\sigma^{2}}{\beta}(1-\nu)+d$, where $d$ is given in (2.29) of Højgaard and Taksar (1999) and

$$
\alpha^{*}(x)=\min \left\{1, \frac{\mu x}{\sigma^{2}(1-\nu)}\right\} .
$$

Asmussen et al. (2000) considered the same problem with excess of loss reinsurance. Højgaard and Taksar (2001) is based on their paper of 1999, where they now assume that the risk reserve is invested in a financial asset with a geometric Brownian motion as price process. The case of a risk free investment into a bond is included. They consider an unrestricted (singular control) dividend payment. The structures of the optimal dividend pay-out rule and reinsurance rule are as in (2.10) and (2.11) respectively.

\section{Convergence Results and Asymptotic Optimality}

The main result of this section is the asymptotic optimality of the optimal feedback rule $\varphi^{*}$ of the controlled diffusion problem. We will use the following notations. As usual we denote by $D_{\mathbb{R}}[0, \infty)$ the set of real-valued functions which are right continuous with lefthand limits. $D_{\mathbb{R}}[0, \infty)$ is endowed with the Skorohod topology. Recall that a sequence of functions $\left\{x^{\gamma}\right\} \subset D_{\mathbb{R}}[0, \infty)$ converges in Skorohod topology for $\gamma \rightarrow \infty$ to $x$ with $x$ continuous if $x_{t}^{\gamma} \rightarrow x_{t}$ uniformly for $t$ in bounded intervals.

\subsection{Preliminary Results}

In this subsection we summarize some preliminary results which will be important for our convergence statements. Proofs of Propositions 3.1 and 3.2 can be found in the appendix. 
Proposition 3.1: For $\gamma>0$ define the process $\left(Z_{t}^{\gamma}\right)$ by

$$
Z_{t}^{\gamma}:=\int_{0}^{t} \alpha_{s}^{\gamma} d M_{s}^{\gamma}
$$

It holds that

a) $\left(Z_{t}^{\gamma}\right)$ is a martingale w.r.t. the filtration $\left\{\mathcal{F}_{t}^{\gamma}\right\}_{t \geq 0}$ for any $\gamma$.

b) $E\left[\left(Z_{t}^{\gamma}\right)^{2}\right] \leq\left(s^{2}-m^{2}\right) \lambda t$ for all $t$ and all $\gamma$.

Next we show the relative compactness of our processes. To ease notation we will define $A_{t}^{\gamma}:=\int_{0}^{t} \alpha_{s}^{\gamma} d s$ and $D_{t}^{\gamma}:=\int_{0}^{t} \delta_{s}^{\gamma} d s$.

Proposition 3.2: The sequence of processes $\left\{\left(A_{t}^{\gamma}, D_{t}^{\gamma}, Z_{t}^{\gamma}\right)\right\}$ is relatively compact in $D_{\mathbb{R}^{3}}[0, \infty)$.

Next we define $C_{L}(\mathbb{R}):=\{x: \mathbb{R} \rightarrow[0,1] \mid x$ is Lipschitz continuous with modul $\leq$ $L\}$. In what follows we consider only reinsurance policies which are feedback rules $\alpha^{\gamma} \in C_{L}(\mathbb{R})$, i.e., the risk reserve is given by

$$
d R_{t}^{\pi^{\gamma}}=\left(\mu \alpha^{\gamma}\left(R_{t}^{\pi^{\gamma}}\right)+\rho\left(R_{t}^{\pi^{\gamma}}\right)-\delta_{t}^{\gamma}\right) d t-\alpha^{\gamma}\left(R_{t}^{\pi^{\gamma}}\right) d M_{t}^{\gamma}
$$

In control problems with infinite time horizon it is quite natural to restrict to feedback controls since the state dynamics are autonomous and time does not play an extra role. Moreover, Schäl (1998) has shown that the optimal control is of feedback type. However, the assumption that $\alpha^{\gamma} \in C_{L}(\mathbb{R})$ is certainly a restriction. We need it to apply Proposition 3.4 for the convergence of stochastic integrals of the type $\int \alpha^{\gamma}\left(X_{t}^{\gamma}\right) d M_{t}^{\gamma}$. As far as the dividend pay-out control $\delta^{\gamma}$ is concerned, we do not need any extra assumption and thus leave it as general as possible. The case of $\delta^{\gamma}$ being a feedback rule $\delta_{t}^{\gamma}=\delta\left(R_{t}^{\pi^{\gamma}}\right)$ is of course covered.

Note that the interest function $\rho$ is also Lipschitz continuous which implies that the SDE has a unique solution $\left(R_{t}^{\pi^{\gamma}}\right)$. By $\Rightarrow$ we denote weak convergence. In what follows we consider some general results. Suppose that $\left\{\left(U_{t}^{\gamma}, Y_{t}^{\gamma}\right)\right\}$ is a sequence of semimartingales with paths in $D_{\mathbb{R} \times \mathbb{R}^{n}}[0, \infty)$ which converge weakly to $\left(U_{t}, Y_{t}\right)$ in Skorohod topology. Further suppose that $f^{\gamma}: \mathbb{R} \rightarrow \mathbb{R}^{n}$ is a sequence of functions with $f^{\gamma} \rightarrow f$ in an appropriate sense. We will investigate under which assumptions solutions of the SDE

$$
d X_{t}^{\gamma}=d U_{t}^{\gamma}+f^{\gamma}\left(X_{t}^{\gamma}\right) d Y_{t}^{\gamma}
$$

converge weakly to a solution of the SDE

$$
d X_{t}=d U_{t}+f\left(X_{t}\right) d Y_{t}
$$


Note that $Y$ and $f$ are vector-valued, i.e. $f\left(X_{t}\right) d Y_{t}=f_{1}\left(X_{t}\right) d Y_{1}(t)+\ldots+f_{n}\left(X_{t}\right) d Y_{n}(t)$. The following two Propositions will be useful. A proof of Proposition 3.3 can be found in Schmidli (1994), Theorem 2. Proposition 3.4 follows from Theorem 2.7 and Proposition 5.1 in Kurtz and Protter (1991).

Proposition 3.3: Let $f: \mathbb{R} \rightarrow \mathbb{R}$ be a Lipschitz-continuous function and $\left\{\left(U_{t}^{\gamma}\right)\right\},\left(U_{t}\right)$ semimartingales in $D_{\mathbb{R}}[0, \infty)$ where $\left(U_{t}\right)$ is continuous. Suppose that $\left(X_{t}^{\gamma}\right)$ is a solution of the $S D E$

$$
d X_{t}^{\gamma}=d U_{t}^{\gamma}+f\left(X_{t}^{\gamma}\right) d t .
$$

If $\left(U_{t}^{\gamma}\right) \Rightarrow\left(U_{t}\right)$ in Skorohod topology, then $\left(X_{t}^{\gamma}\right) \Rightarrow\left(X_{t}\right)$ in Skorohod topology and $\left(X_{t}\right)$ satisfies the $S D E$

$$
d X_{t}=d U_{t}+f\left(X_{t}\right) d t
$$

For a finite variation process $\left(L_{t}\right)$ define $T_{t}(L):=\sup _{0 \leq t_{1} \leq \ldots \leq t_{n} \leq t} \sum_{i}\left|L_{t_{i+1}}-L_{t_{i}}\right|$ where the supremum is taken over all partitions of the interval $[0, t]$.

Proposition 3.4: For each $\gamma$ let $f, f^{\gamma}: \mathbb{R} \rightarrow \mathbb{R}^{n}$ and $\left\{\left(Y_{t}^{\gamma}, U_{t}^{\gamma}, X_{t}^{\gamma}, f^{\gamma}\left(X_{t}^{\gamma}\right)\right)\right\}$ be a sequence in $D_{\mathbb{R}^{3+n}}[0, \infty)$ with $\left(Y_{t}^{\gamma}, U_{t}^{\gamma}, X_{t}^{\gamma}, f^{\gamma}\left(X_{t}^{\gamma}\right)\right) \Rightarrow\left(Y_{t}, U_{t}, X_{t}, f\left(X_{t}\right)\right)$ in Skorohod topology where $\left(Y_{t}^{\gamma}, U_{t}^{\gamma}\right)$ and $\left(Y_{t}, U_{t}\right)$ are semimartingales. Suppose further that the sequence satisfies the SDE

$$
d X_{t}^{\gamma}=d U_{t}^{\gamma}+f^{\gamma}\left(X_{t}^{\gamma}\right) d Y_{t}^{\gamma}
$$

and that $Y_{t}^{\gamma}=M_{t}^{\gamma}+L_{t}^{\gamma}$, where $\left(M_{t}^{\gamma}\right)$ is a local $\left\{\mathcal{F}_{t}^{\gamma}\right\}$-martingale and $\left(L_{t}^{\gamma}\right)$ is an $\left\{\mathcal{F}_{t}^{\gamma}\right\}$ adapted finite variation process. If $\sup _{\gamma} E\left[\left[M^{\gamma}\right]_{t}+T_{t}\left(L^{\gamma}\right)\right]<\infty$ is satisfied, then the limit solves the SDE

$$
d X_{t}=d U_{t}+f\left(X_{t}\right) d Y_{t}
$$

\subsection{The Main Convergence Results}

Applying the Propositions of section 3.1 we will first show that the sequence of scaled risk reserve and action processes has convergent subsequences and every possible limit satisfies the SDE of the controlled diffusion process with a suitable policy.

Theorem 3.5: Let $\left\{\pi^{\gamma}\right\}$ be any sequence of policies and $A_{t}^{\gamma}:=\int_{0}^{t} \alpha^{\gamma}\left(R_{s}^{\pi^{\gamma}}\right) d s$. Every sequence of processes $\left\{\left(A_{t}^{\gamma}, D_{t}^{\gamma}, M_{t}^{\gamma}, R_{t}^{\pi^{\gamma}}\right)\right\}$ has a further subsequence, indexed $\left\{\gamma_{n}\right\}$, such that

$$
\left(A_{t}^{\gamma_{n}}, D_{t}^{\gamma_{n}}, M_{t}^{\gamma_{n}}, R_{t}^{\pi^{\gamma_{n}}}\right) \Rightarrow\left(A_{t}, D_{t}, \sigma B_{t}, R_{t}^{\pi}\right)
$$


in Skorohod topology and the limit satisfies $A_{t}=\int_{0}^{t} \alpha\left(R_{s}^{\pi}\right) d s$ with $\alpha(.) \in[0,1], D_{t}=\int_{0}^{t} \delta_{s} d s$ with $\delta_{s} \in[0, \Delta]$, and

$$
d R_{t}^{\pi}=\left(\mu \alpha\left(R_{t}^{\pi}\right)+\rho\left(R_{t}^{\pi}\right)-\delta_{t}\right) d t-\sigma \alpha\left(R_{t}^{\pi}\right) d B_{t} .
$$

Moreover, there exists a filtration $\left\{\mathcal{G}_{t}\right\}_{t \geq 0}$ such that the processes $\left(A_{t}, D_{t}, B_{t}, R_{t}^{\pi}\right)$ are adapted w.r.t. this filtration and $\left(B_{t}\right)$ is a standard Brownian motion w.r.t. $\left\{\mathcal{G}_{t}\right\}_{t \geq 0}$.

Proof: From Proposition 3.2 we know that the sequence of processes $\left\{\left(A_{t}^{\gamma}, D_{t}^{\gamma}, Z_{t}^{\gamma}\right)\right\}$ is relatively compact and thus there exists a convergent subsequence indexed by $\left\{\gamma_{n}\right\}$ (for notational convenience however we will still denote it by $\{\gamma\}$ ). Since the interest function $\rho$ is Lipschitz continuous, Proposition 3.3 implies that $\left\{\left(R_{t}^{\pi^{\gamma}}\right)\right\}$ also converges. Last but not least it is well-known that $\left(M_{t}^{\gamma}\right) \Rightarrow\left(\sigma B_{t}\right)$ (see e.g. Grandell (1977), Harrison (1977)), where $\left(B_{t}\right)$ is a standard Brownian motion w.r.t. the natural filtration $\left\{\sigma\left(B_{s}, s \leq t\right)\right\}_{t \geq 0}$. Thus, it follows that the sequence converges. Next we will show that $\left(B_{t}\right)$ is a Brownian motion w.r.t. the filtration $\left\{\mathcal{G}_{t}\right\}_{t \geq 0}$, where $\mathcal{G}_{t}:=\sigma\left(A_{s}, D_{s}, B_{s}, R_{s}^{\pi}, s \leq t\right)$. Trivially all limiting processes are $\left\{\mathcal{G}_{t}\right\}_{t \geq 0}$-adapted. To this end let $H: \mathbb{R}^{4 n} \rightarrow \mathbb{R}$ be any bounded continuous function and $0<t_{1}<\ldots<t_{n}<t$. Then

$$
\begin{aligned}
& E\left[H\left(A_{t_{i}}^{\gamma}, D_{t_{i}}^{\gamma}, M_{t_{i}}^{\gamma}, R_{t_{i}}^{\pi^{\gamma}}, i=1, \ldots, n\right) \times\left(M_{t+h}^{\gamma}-M_{t}^{\gamma}\right)\right] \\
= & E\left[H\left(A_{t_{i}}^{\gamma}, D_{t_{i}}^{\gamma}, M_{t_{i}}^{\gamma}, R_{t_{i}}^{\pi^{\gamma}}, i=1, \ldots, n\right)\right] \times E\left[\left(M_{t+h}^{\gamma}-M_{t}^{\gamma}\right)\right]=0
\end{aligned}
$$

Taking limit $\gamma \rightarrow \infty$ yields

$$
E\left[H\left(A_{t_{i}}, D_{t_{i}}, B_{t_{i}}, R_{t_{i}}^{\pi}, i=1, \ldots, n\right) \times\left(B_{t+h}-B_{t}\right)\right]=0 .
$$

Note that $\left(M_{t+h}^{\gamma}-M_{t}^{\gamma}\right)$ is uniformly integrable since $\sup _{\gamma} E\left[\left(M_{t+h}^{\gamma}-M_{t}^{\gamma}\right)^{2}\right] \leq$ $\left(s^{2}-m^{2}\right) \lambda h$. In a similar way one can show that

$$
E\left[H\left(A_{t_{i}}, D_{t_{i}}, B_{t_{i}}, R_{t_{i}}^{\pi}, i=1, \ldots, n\right) \times\left\{\left(B_{t+h}-B_{t}\right)^{2}-\sigma^{2} h\right\}\right]=0 .
$$

Thus, $\left(B_{t}\right)$ is a Brownian motion w.r.t. $\left\{\mathcal{G}_{t}\right\}_{t \geq 0}$ (see e.g. Kushner (2001) p. 50 or Liptser and Shiryaev (1977) Theorem 4.1).

It remains now to characterize the limit. Since $\delta_{t}^{\gamma} \in[0, \Delta]$ for all $\gamma$ and $t$, the limit of $\left(D_{t}^{\gamma}\right)$ can be written as $D_{t}=\int_{0}^{t} \delta_{s} d s$ with $\delta_{t} \in[0, \Delta]$ for all $t$. Since $\alpha^{\gamma} \in C_{L}(\mathbb{R})$ for all $\gamma$, the sequence $\left\{\alpha^{\gamma}\right\}$ is equicontinuous (and trivially pointwise bounded). According to the theorem of Arzela and Ascoli a subsequence $\left\{\gamma_{n}\right\}$ can be chosen such that $\alpha^{\gamma_{n}}(x) \rightarrow \alpha(x)$ uniformly on compact sets (the sequence will still be denoted by $\{\gamma\}$ ). Now suppose $\left\{x^{\gamma}\right\} \subset D_{\mathbb{R}}[0, \infty)$ and $x^{\gamma} \rightarrow x$ in Skorohod topology, with $x$ continuous. We will show that $\alpha^{\gamma}\left(x_{t}^{\gamma}\right) \rightarrow \alpha\left(x_{t}\right)$ in Skorohod topology. First we obtain

$$
\sup _{0 \leq t \leq T}\left|\alpha^{\gamma}\left(x_{t}^{\gamma}\right)-\alpha\left(x_{t}\right)\right| \leq \sup _{0 \leq t \leq T}\left|\alpha^{\gamma}\left(x_{t}^{\gamma}\right)-\alpha^{\gamma}\left(x_{t}\right)\right|+\sup _{0 \leq t \leq T}\left|\alpha^{\gamma}\left(x_{t}\right)-\alpha\left(x_{t}\right)\right| .
$$


For $\gamma \rightarrow \infty$ the first term vanishes since $\left|\alpha^{\gamma}\left(x_{t}^{\gamma}\right)-\alpha^{\gamma}\left(x_{t}\right)\right| \leq L\left|x_{t}^{\gamma}-x_{t}\right|$ and $x^{\gamma} \rightarrow x$ uniformly on compact sets by assumption. For the second term we know that $x_{t} \in$ $\left[\min _{t \in[0, T]} x_{t}, \max _{t \in[0, T]} x_{t}\right]$ for $t \in[0, T]$. Since $\alpha^{\gamma} \rightarrow \alpha$ uniformly on compact sets, the second term also vanishes and the statement is shown. Thus, we have now that $\left(\alpha^{\gamma}\left(R_{t}^{\pi^{\gamma}}\right)\right) \Rightarrow\left(\alpha\left(R_{t}^{\pi}\right)\right)$. In particular it also holds that $\left(\rho\left(R_{t}^{\pi^{\gamma}}\right)\right) \Rightarrow\left(\rho\left(R_{t}^{\pi}\right)\right)$. Finally note that due to Proposition 3.1

$$
\sup _{\gamma} E\left[\left[M^{\gamma}\right]_{t}\right]=\sup _{\gamma} E\left[\left(M_{t}^{\gamma}\right)^{2}\right] \leq\left(s^{2}-m^{2}\right) \lambda t
$$

and for $X_{t}=t, T_{t}(X)=t$. Applying Proposition 3.4 yields the result.

The next theorem shows that the value function of the controlled diffusion problem provides an asymptotic upper bound for the value functions of the piecewise deterministic Markov decision processes under diffusion scaling.

Theorem 3.6: For every $x$ it holds that

$$
\limsup _{\gamma \rightarrow \infty} V^{\gamma}(x) \leq W(x)
$$

Proof: Let $\left\{\pi^{\gamma}\right\}$ be an arbitrary sequence of policies for the scaled piecewise deterministic risk reserve process. In what follows we suppose that $\{\gamma\}$ is a subsequence which yields convergence in Theorem 3.5. Since $T_{t}\left(D^{\gamma}\right) \leq \Delta t$, weak convergence $\left(D_{t}^{\gamma}\right) \Rightarrow\left(D_{t}\right)$ implies according to Proposition 3.4 that

$$
\left(\int_{0}^{t} e^{-\beta s} d D_{s}^{\gamma}\right) \Rightarrow\left(\int_{0}^{t} e^{-\beta s} d D_{s}\right)
$$

for $\gamma \rightarrow \infty$ and $\left(\int_{0}^{t} e^{-\beta s} d D_{s}^{\gamma}\right)$ is bounded by $\frac{\Delta}{\beta}$ in $t$ and $\gamma$.

Next we have to deal with the ruin time $\tau(\cdot)$. Note that under any policy $\pi$ the trajectory of the diffusion risk reserve $\left(R_{t}^{\pi}\right)$ when hitting zero at time $t^{*}=\inf \{t \geq$ $\left.0 \mid R_{t}^{\pi} \leq 0\right\}$ can only show the following two behaviors with probability 1 : it is absorbed at zero (in this case $\alpha(0)=\delta(0)=0$ ) and no further dividend is payed out or for any $\epsilon>0$ there exists a $t \in\left[t^{*}, t^{*}+\epsilon\right]$ such that $R_{t}^{\pi}<0$. No reflection can occur due to the properties of the Brownian sample paths. From the Skorohod theorem we know that it is possible to construct a probability space such that

$$
\left(R_{t}^{\pi^{\gamma}}, \int_{0}^{t} e^{-\beta s} d D_{s}^{\gamma}\right) \rightarrow\left(R_{t}^{\pi}, \int_{0}^{t} e^{-\beta s} d D_{s}\right)
$$

almost surely and uniformly on compact sets for $\gamma \rightarrow \infty$. We will now show that

$$
\int_{0}^{\tau\left(R^{\pi^{\gamma}}\right)} e^{-\beta s} d D_{s}^{\gamma} \rightarrow \int_{0}^{\tau\left(R^{\pi}\right)} e^{-\beta s} d D_{s}
$$


almost surely for $\gamma \rightarrow \infty$. Let us first fix a realisation $\omega$ where $\left(R_{t}^{\pi}(\omega)\right)$ is absorbed at zero at time $t^{*}$. Then we obtain

$$
\liminf _{\gamma \rightarrow \infty} \tau\left(R^{\pi^{\gamma}}(\omega)\right) \geq t^{*}
$$

because of the following observation: let us first skip $\omega$ to ease notation. Suppose now that $\liminf _{\gamma \rightarrow \infty} \tau\left(R^{\pi^{\gamma}}\right)=: \hat{t}<t^{*}$. Since $R_{t}^{\pi}$ is continuous and due to the definition of $t^{*}$ we have $0<\underline{R}:=\min _{t \in[0, \hat{t}]} R_{t}^{\pi}$. However, we can choose now $\hat{\gamma}$ large enough such that $\sup _{t \in[0, \hat{t}]}\left|R_{t}^{\pi^{\gamma}}-R_{t}^{\pi}\right|<\underline{R}$ for all $\gamma \geq \hat{\gamma}$ which implies that $\tau\left(R^{\pi^{\gamma}}\right)>\hat{t}$ for all $\gamma \geq \hat{\gamma}$. This is of course a contradiction to our assumption and (3.15) holds.

Now we proceed as follows. Since

$$
\int_{0}^{\tau\left(R^{\pi^{\gamma}}\right)} e^{-\beta s} d D_{s}^{\gamma}=\int_{0}^{t^{*}} e^{-\beta s} d D_{s}^{\gamma}+\int_{t^{*}}^{\tau\left(R^{\pi^{\gamma}}\right)} e^{-\beta s} d D_{s}^{\gamma}
$$

and

$$
\int_{0}^{\tau\left(R^{\pi}\right)} e^{-\beta s} d D_{s}=\int_{0}^{t^{*}} e^{-\beta s} d D_{s}
$$

it remains to show that $\int_{t^{*}}^{\tau\left(R^{\pi^{\gamma}}\right)} e^{-\beta s} d D_{s}^{\gamma} \rightarrow 0$ almost surely in this case. In view of (3.15) we restrict to the case $\tau\left(R^{\pi^{\gamma}}\right) \geq t^{*}$ for all $\gamma$. Due to our assumption we have for any $T>t^{*}$ that if $\gamma$ is large enough

$$
\begin{aligned}
\int_{t^{*}}^{\tau\left(R^{\pi^{\gamma}}\right)} e^{-\beta s} d D_{s}^{\gamma} & \leq \int_{t^{*}}^{\infty} e^{-\beta s} d D_{s}^{\gamma}=\int_{t^{*}}^{T} e^{-\beta s} d D_{s}^{\gamma}+\int_{T}^{\infty} e^{-\beta s} d D_{s}^{\gamma} \\
& \leq \frac{1}{T}+\frac{\Delta}{\beta} e^{-\beta T} .
\end{aligned}
$$

In order to see the second inequality for the first integral note that from our convergence result $\int_{t^{*}}^{T} e^{-\beta s} d D_{s}^{\gamma} \rightarrow 0$ for $\gamma \rightarrow \infty$. Thus, for fixed $T>t^{*}$, there exists a $\gamma(T)$ large enough such that $\int_{t^{*}}^{T} e^{-\beta s} d D_{s}^{\gamma}<\frac{1}{T}$ for all $\gamma \geq \gamma(T)$. Letting $T \rightarrow \infty$ yields our statement.

Let us next fix a realisation $\omega$ where $\left(R_{t}^{\pi}(\omega)\right)$ is not absorbed (which includes the case $\left.\tau\left(R^{\pi}\right)=\infty\right)$. For $x \in D_{\mathbb{R}}[0, \infty)$ consider the mapping $x \mapsto \tau(x):=\inf \left\{t \geq 0 \mid x_{t}<0\right\}$. It is well-known that $\tau(x)$ is continuous at those functions $x$ with $x=R^{\pi}(\omega)$ (see e.g. Kushner and Dupuis (1994) p. 261, Schmidli (1994) Proposition 2). Thus, altogether $\left(R_{t}^{\pi^{\gamma}}, D_{t}^{\gamma}\right) \Rightarrow\left(R_{t}^{\pi}, D_{t}\right)$ in $D_{\mathbb{R}^{2}}[0, \infty)$ implies by bounded convergence that

$$
\lim _{\gamma \rightarrow \infty} V_{\pi^{\gamma}}^{\gamma}(x)=\lim _{\gamma \rightarrow \infty} E\left[\int_{0}^{\tau\left(R^{\pi^{\gamma}}\right)} e^{-\beta s} d D_{s}^{\gamma}\right]=E\left[\int_{0}^{\tau\left(R^{\pi}\right)} e^{-\beta s} d D_{s}\right]=W_{\pi}(x) .
$$

Since every sequence $\left\{\left(A_{t}^{\gamma}, D_{t}^{\gamma}, M_{t}^{\gamma}, R_{t}^{\pi^{\gamma}}\right)\right\}$ has a converging subsequence according to Theorem 3.5 and the limit satisfies the SDE

$$
d R_{t}^{\pi}=\left(\mu \alpha\left(R_{t}^{\pi}\right)+\rho\left(R_{t}^{\pi}\right)\right) d t-d D_{t}-\sigma \alpha\left(R_{t}^{\pi}\right) d B_{t}
$$

and is thus a controlled diffusion with policy $\pi_{t}=\left(\alpha\left(R_{t}^{\pi}\right), \delta_{t}\right), D_{t}=\int_{0}^{t} \delta_{s} d s$, we obtain

$$
\limsup _{\gamma \rightarrow \infty} V_{\pi^{\gamma}}^{\gamma}(x) \leq W(x) .
$$


This statement also holds for the sequence of optimal policies $\left\{\pi^{* \gamma}\right\}$ of the piecewise deterministic Markov decision processes and the theorem is shown.

Last but not least we show that an optimal feedback rule $\varphi^{*}$ for the diffusion problem which is of the structure given in (2.8)-(2.11) achieves the upper bound $W(x)$ as $\gamma$ tends to infinity. Note that the feedback rule $\varphi^{*}$ is always admissible in the piecewise deterministic setting. For $\beta_{0}=0$ the optimality of $\varphi^{*}$ given in (2.8)-(2.11) has been shown in Højgaard and Taksar (1999). For $\beta_{0}>0$ the problem has been treated in Højgaard and Taksar (2001), however with unbounded dividend rate $(\Delta=\infty)$.

Theorem 3.7: Suppose the optimal feedback rule $\varphi^{*}$ for the controlled diffusion is of the type given in (2.8)-(2.11). Then $\varphi^{*}$ is asymptotically optimal, i.e.

$$
\lim _{\gamma \rightarrow \infty} V_{\varphi^{*}}^{\gamma}(x)=W(x) .
$$

Proof: Under $\varphi^{*}$ the scaled risk reserve process is given by the SDE

$$
d R_{t}^{\varphi^{*}, \gamma}=\left(\mu \alpha^{*}\left(R_{t}^{\varphi^{*}, \gamma}\right)+\rho\left(R_{t}^{\varphi^{*}, \gamma}\right)-\delta^{*}\left(R_{t}^{\varphi^{*}, \gamma}\right)\right) d t-\alpha\left(R_{t}^{\varphi^{*}, \gamma}\right) d M_{t}^{\gamma} .
$$

As in the proof of Theorem 3.5 it can be shown that every sequence $\left\{\left(R_{t}^{\varphi^{*}, \gamma}\right)\right\}$ has a further subsequence indexed $\left\{\gamma_{n}\right\}$ such that $\left(R_{t}^{\varphi^{*}, \gamma_{n}}\right) \Rightarrow\left(R_{t}^{\varphi^{*}}\right)$. We will now show that

$$
\left(D_{t}^{* \gamma_{n}}\right):=\left(\int_{0}^{t} \delta^{*}\left(R_{s}^{\varphi^{*}, \gamma_{n}}\right) d s\right) \Rightarrow\left(\int_{0}^{t} \delta^{*}\left(R_{s}^{\varphi^{*}}\right) d s\right)=:\left(D_{t}^{*}\right) .
$$

Note that $\delta^{*}$ is not continuous. Since, however, $\alpha^{*}$ is Lipschitz-continuous (3.17) implies that every limit $\left(R_{t}^{\varphi^{*}}\right)$ of a convergent subsequence satisfies the SDE

$$
d R_{t}^{\varphi^{*}}=\left(\mu \alpha^{*}\left(R_{t}^{\varphi^{*}}\right)+\rho\left(R_{t}^{\varphi^{*}}\right)-\delta^{*}\left(R_{t}^{\varphi^{*}}\right)\right) d t-\sigma \alpha^{*}\left(R_{t}^{\varphi^{*}}\right) d B_{t} .
$$

Thus, the sequence $\left\{\left(R_{t}^{\varphi^{*}, \gamma}\right)\right\}$ converges and the limit satisfies (3.18). As in Theorem 3.6 it finally follows that

$$
\lim _{\gamma \rightarrow \infty} V_{\varphi^{*}}^{\gamma}(x)=\lim _{\gamma \rightarrow \infty} E\left[\int_{0}^{\tau\left(R^{\varphi^{*}, \gamma}\right)} e^{-\beta s} d D_{s}^{* \gamma}\right]=E\left[\int_{0}^{\tau\left(R^{\varphi^{*}}\right)} e^{-\beta s} d D_{s}^{*}\right]=W_{\varphi^{*}}(x)=W(x)
$$

and the statement is shown.

As far as (3.17) is concerned we know from the Skorohod theorem that it is possible to construct a probability space such that $R_{t}^{\varphi^{*}, \gamma} \rightarrow R_{t}^{\varphi^{*}}$ almost surely and uniformly on compact sets. Choose $\gamma$ large enough to obtain $\sup _{0 \leq s \leq t}\left|R_{s}^{\varphi^{*}, \gamma}-R_{s}^{\varphi^{*}}\right| \leq \varepsilon$ for $\varepsilon>0$. Hence (note that $m$ is the parameter appearing in the optimal dividend pay-out feedback rule $\left.\delta^{*}\right)$

$$
\left|D_{t}^{* \gamma}-D_{t}^{*}\right| \leq \int_{0}^{t}\left|\delta^{*}\left(R_{s}^{\varphi^{*}, \gamma}\right)-\delta^{*}\left(R_{s}^{\varphi^{*}}\right)\right| d s \leq \Delta \int_{0}^{t} I_{\left[R_{s}^{\varphi^{*}} \in[m-\varepsilon, m+\varepsilon]\right]} d s
$$


For $\varepsilon \rightarrow 0$ the right-hand-side converges almost surely to $\Delta \int_{0}^{t} I_{\left[R_{s}^{\varphi^{*}}=m\right]} d s$. Since

$$
E\left[\int_{0}^{t} I_{\left[R_{s}^{\varphi^{*}}=m\right]}\right] d s=\int_{0}^{t} P\left(R_{s}^{\varphi^{*}}=m\right) d s=0,
$$

we have $I_{\left[R_{s}^{\varphi^{*}}=m\right]}=0$ a.s. for $0 \leq s \leq t$. This observation concludes the proof.

\section{Economic Interpretation and Numerical Results}

For the following numerical example, the dividend pay-out problem has been considered with data $\lambda=0.5, F \sim \exp (1), c=1.5, \beta=0.1, \beta_{0}=0, \Delta=0.5$. The solution of the controlled diffusion problem gives an optimal dividend pay-out threshold of $m=1.226$ and the optimal value function $W(x)$ plotted in figure 3.1. Obviously $\frac{\Delta}{\beta}=5$ is an upper bound for $W(x)$ which can be attained if it is possible to pay out the maximal dividend rate at all times. We have now used this control for the original piecewise deterministic Markov decision process. The corresponding value functions $V_{\varphi^{*}}^{\gamma}(x)$ are plotted in figure 3.1 for different values of $\gamma$. As proved in Theorem 3.7, $V_{\varphi^{*}}^{\gamma}(x)$ appears to converges pointwise to $W(x)$ as $\gamma$ becomes larger.

What is now the economical relevance of our results? Of course the original dividend pay-out model itself is very simple. As such, one would expect to obtain a simple policy which can serve as a "rule of thumb" and shows the important structural properties. This is not the case as we have outlined in the introduction. However, when the insurance company is large (which means that $\gamma$ is large) Theorem 3.6 and Theorem 3.7 imply that the simple feedback rule $\varphi^{*}$ obtained from the controlled diffusion problem is $\epsilon$ optimal since $\left|V_{\varphi^{*}}^{\gamma}(x)-V^{\gamma}(x)\right|$ is small. For practical purposes this approximation seems to be good enough. Intuitively one might think that a statement like this is clear. Mathematically however, the question is delicate and has only been shown for Lipschitzcontinuous reinsurance rules. It remains open whether a similar statement is true in a more general setting. Finally, the problem in the diffusion setting has another advantage: only the first and second moment of the claim size distribution are needed. The precise distribution of the claims is not necessary to know. On the negative side the diffusion approximation rules out claims with infinite second moment.

\section{Appendix}

Proof of Proposition 3.1: The proof is similar to the one of Asmussen (1987) for Lemma 3.4 (p. 138). First define $Y_{t}^{\gamma}:=Y_{n}^{\gamma}$ if $T_{n}^{\gamma} \leq t<T_{n+1}^{\gamma}$, where $\left\{T_{n}^{\gamma}\right\}$ is the sequence of jump time points of the Poisson process $\left(N_{t}^{\gamma}\right)$ with intensity $\lambda \cdot \gamma$. For natural numbers $k \in \mathbb{N}$ and $l=0,1, \ldots, k$ let $t_{l}:=\frac{l}{k} t$ where $t>0$. Define

$$
Z_{(k, l)}^{\gamma}(t):=\alpha^{\gamma}\left(t_{l-1}\right) Y^{\gamma}\left(t_{l}\right) I_{\left[\left(N^{\gamma}\left(t_{l}\right)-N^{\gamma}\left(t_{l-1}\right)\right)>0\right]}-\alpha^{\gamma}\left(t_{l-1}\right) \frac{m}{\sqrt{\gamma}}\left(1-e^{-\frac{\lambda t \gamma}{k}}\right)
$$


and

$$
Z_{k}^{\gamma}(t):=\sum_{l=1}^{k} Z_{(k, l)}^{\gamma}(t)
$$

Then it is rather easy to see that $Z_{k}^{\gamma}(t) \rightarrow Z_{t}^{\gamma}$ almost surely for $k \rightarrow \infty$ (note that $k\left(1-e^{-\frac{\lambda}{k}}\right) \rightarrow \lambda$ for $\left.k \rightarrow \infty\right)$. Next it holds that

$$
E\left[Z_{(k, l)}^{\gamma}(t) \mid \mathcal{F}_{t_{l-1}}^{\gamma}\right]=\alpha^{\gamma}\left(t_{l-1}\right)\left(\frac{m}{\sqrt{\gamma}}\left(1-e^{-\frac{\lambda t \gamma}{k}}\right)-\frac{m}{\sqrt{\gamma}}\left(1-e^{-\frac{\lambda t \gamma}{k}}\right)\right)=0 .
$$

Since $\left|Z_{k}^{\gamma}(t)\right|$ is bounded by $\sum_{n=1}^{N^{\gamma}(t)} Y_{n}^{\gamma}$ plus a constant, the last result implies that $\left(Z_{t}^{\gamma}\right)$ is a martingale w.r.t. $\left\{\mathcal{F}_{t}^{\gamma}\right\}_{t \geq 0}$ and part a) is shown. For part b) we obtain

$$
\begin{aligned}
E\left[\left(Z_{t}^{\gamma}\right)^{2}\right] & =\lim _{k \rightarrow \infty} E\left[\left(Z_{k}^{\gamma}(t)\right)^{2}\right]=\lim _{k \rightarrow \infty} \sum_{l=1}^{k} E\left[\left(Z_{(k, l)}^{\gamma}(t)\right)^{2}\right] \leq \\
& \leq \lim _{k \rightarrow \infty} k \cdot \operatorname{Var}\left[Y^{\gamma}(t / k) I_{\left[N^{\gamma}\left(\frac{t}{k}\right)>0\right]}\right]= \\
& =\lim _{k \rightarrow \infty} k\left(\frac{s^{2}-m^{2}}{\gamma}\left(1-e^{-\frac{\lambda t \gamma}{k}}\right)-\frac{m^{2}}{\gamma}\left(1-e^{-\frac{\lambda t \gamma}{k}}\right)\right) \leq\left(s^{2}-m^{2}\right) \lambda t
\end{aligned}
$$

and the assertion follows.

Proof of Proposition 3.2: According to Proposition 3.2.4 in Ethier and Kurtz (1986) the statement can be shown separately for each sequence of processes. A well-known criterion for relative compactness of a sequence of processes $\left\{\left(X_{t}^{\gamma}\right)\right\}$ in $D_{\mathbb{R}}[0, \infty)$ is as follows (see e.g. Kushner and Dupuis (1992) Theorem 2.1)

(i) $\lim _{K \rightarrow \infty} \sup _{\gamma} P\left(\left|X_{t}^{\gamma}\right| \geq K\right)=0$ for all $t \geq 0$.

(ii) $\lim _{h \rightarrow 0} \lim \sup _{\gamma \rightarrow \infty} \sup _{t \leq T} E\left[\min \left\{1,\left|X_{t+h}^{\gamma}-X_{t}^{\gamma}\right|\right\}\right]=0$.

The sequences $\left\{\left(A_{t}^{\gamma}\right)\right\}$ and $\left\{\left(D_{t}^{\gamma}\right)\right\}$ are trivially relatively compact since for all $\gamma$

$$
\left|A_{t+h}^{\gamma}-A_{t}^{\gamma}\right| \leq h \text { a.s., } \quad \text { and }\left|D_{t+h}^{\gamma}-D_{t}^{\gamma}\right| \leq h \Delta \text { a.s. }
$$

For the last sequence we obtain with the Chebyshev inequality and Proposition 3.1 that

$$
P\left(\left|Z_{t}^{\gamma}\right| \geq K\right) \leq \frac{1}{K^{2}} E\left[\left(Z_{t}^{\gamma}\right)^{2}\right] \leq \frac{1}{K^{2}}\left(s^{2}-m^{2}\right) \lambda t
$$

which implies (i). For (ii) note that again with Proposition 3.1

$$
\left(E\left[\left|Z_{t+h}^{\gamma}-Z_{t}^{\gamma}\right|\right]\right)^{2} \leq E\left[\left(Z_{t+h}^{\gamma}-Z_{t}^{\gamma}\right)^{2}\right] \leq\left(s^{2}-m^{2}\right) \lambda h
$$

and (ii) follows which implies the result. 


\section{REFERENCES}

Asmussen, S. (1987): Applied probability and queues. Chichester: John Wiley \& Sons.

Asmussen, S., And M. TAKsar (1997): Controlled diffusion models for optimal dividend pay-out. Insurance: Math. $\&$ Economics 20, 1-15.

Asmussen, S., B. HøjgaArd, And M. Taksar (2000): Optimal risk control and dividend distribution policies. Example of excess-of loss reinsurance for an insurance corporation. Finance and Stochastics 4, 299-324.

Borch, K. (1967): The theory of risk. J. Royal Statist. Soc. B 29, 432-452.

Borch, K. (1969): The capital structure of a firm. Swedish J. Econ. 71, 1-13.

Browne, S. (1995): Optimal investment policies for a firm with random risk process: exponential utility and minimizing the probability of ruin. Math. Operations Res. 20, 937-958.

Browne, S. (1997): Survival and growth with liability: optimal portfolio strategies in continuous time. Math. Operations Res. 22, 468-493.

Bühlmann, H. (1970): Mathematical Methods in Risk Theory. Berlin: SpringerVerlag.

Dassios, A., And P. Embrechts (1989): Martingales and insurance risk. Commun. Statist. - Stochastic Models 5, 181-217.

Davis, M.H.A. (1993): Markov models and optimization. London: Chapman \& Hall.

DE FinetTi, B. (1957): Su un'impostazione alternativa dell teoria colletiva del rischio. Transactions of the 15th International Congress of Actuaries, New York, 2, 433443.

Embrechts, P., AND H. Schmidli (1994): Ruin estimation for a general insurance risk model. Adv. in Appl. Probab. 26, 404-422.

Ethier, S.N., And T.G. KurTz (1986): Markov processes. New York: John Wiley \& Sons.

Fleming, W.H., AND R.W. Rishel (1975): Deterministic and stochastic optimal control. New York: Springer-Verlag.

Fleming, W.H., AND H.M. Soner (1993): Controlled Markov processes and viscosity solutions. New York: Springer-Verlag.

Gerber, H.U. (1972): Games of economic survival with discrete- and continuousincome processes. Operations Research 20, 37-45. 
Gerber, H.U. (1979): An introduction to mathematical risk theory. S.S. Huebner Foundation Monographs, University of Pennsylvania.

Grandell, J. (1977): A class of approximations of ruin probabilities. Scand. Actuarial J., Suppliment 37-52.

Grandell, J. (1991): Aspects of risk theory. New York: Springer-Verlag.

Harrison, J.M. (1977): Ruin problems with compounding assets. Stochastic Processes Appl. 5, 67-79.

Hipp, C., And M. Vogt (2001): Optimal dynamic XL reinsurance. Preprint, University of Karlsruhe.

HøJgaARD, B., AND M. TAKsar (1998): Optimal proportional reinsurance policies for diffusion models. Scand. Actuarial J. 2, 166-180.

HøjgaARD, B., AND M. TAKSAR (1999): Controlling risk exposure and dividend payout schemes: insurance company example. Math. Finance 9(2), 153-182.

HøjgaARD, B., AND M. TAKsar (2001): Optimal risk control for a large corporation in the presence of return on investments. Finance and Stochastics 5, 527-547.

KRYlov, N.V. (1980): Controlled diffusion processes. New York: Springer-Verlag.

Kurtz, T.G., And P. Protter (1991): Weak limit theorems for stochastic integrals and stochastic differential equations. Ann. Appl. Probab. 19(3), 1035-1070.

Kushner, H.J., AND P.G. Dupuis (1992): Numerical methods for stochastic control problems in continuous time. New York: Springer-Verlag.

Kushner, H.J. (2001): Heavy traffic analysis of controlled queueing and communication networks. New York: Springer-Verlag.

Liptser, R., And A.N. Shiryaev (1977): Statistics of random processes. Berlin and New York: Springer-Verlag.

Maglaras, C. (2000): Discrete-review policies for scheduling stochastic networks: trajectory tracking and fluid-scale asymptotic optimality. Ann. Appl. Probab. 10, 897-929.

Miller, M.H., AND F. Modigliani (1961): Dividend policy, growth and valuation of shares. J. Business 34, 411-433.

RAdner, R., And L. Shepp (1996): Risk vs. Profit potential: a model for corporate strategy. J. Econ. Dynam. Control, 1373-1393.

SCḦ̈L, M. (1998): On piecewise deterministic Markov control processes: control of jumps and of risk processes in insurance. Insurance Math.\& Econom. 22, 75-91. 
SCHÄL, M. (2002): On the first order Bellman equation in reinsurance and investment control problems. Preprint, University of Bonn.

SCHMIDLI, H. (1994): Diffusion approximation for a risk process with the possibility of borrowing and investment. Commun. Statist.- Stochastic Models 10(2), 365-388.

SCHMidLi, H. (2001): Optimal proportional reinsurance policies in a dynamic setting. Scand. Actuarial J. 1, 55-68.

SCHMIDLI, H. (2002): On minimising the ruin probability by investment and reinsurance. Preprint, University of Aarhus.

Sethi, S.P. (1996): When does the share price equal the present value of future dividends? A modified dividend approach. Econ. Theory 8, 307-319.

Sethi, S.P., N.A. Derzko AND J. Lehoczky (1991): A stochastic extension of Miller Modigliani framework. Math. Finance 1(4), 57-76. 


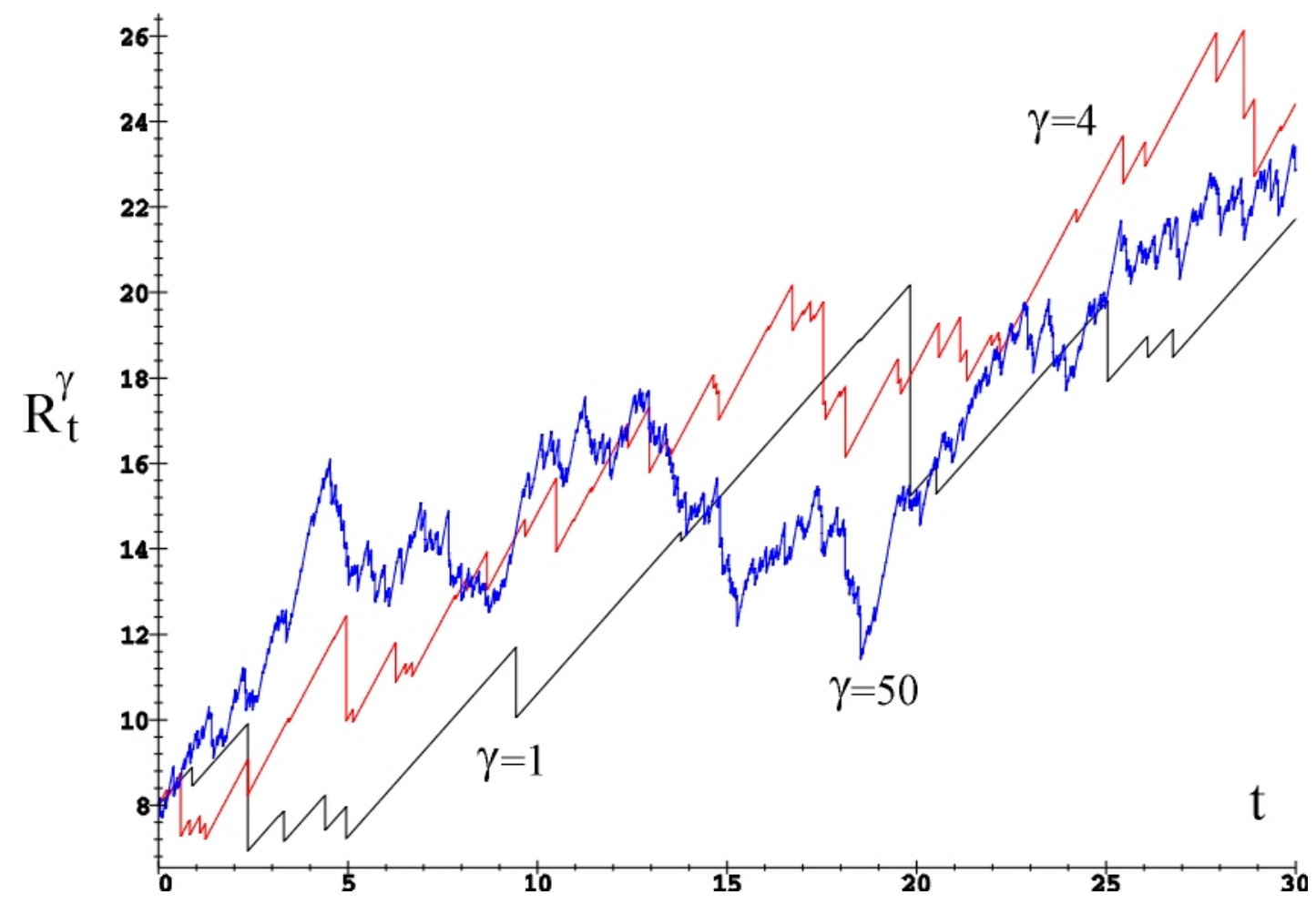

fig 1.1.: $\left(R_{t}^{\gamma}\right)$ for $\gamma=1,4,50$ and data $\lambda=0.5, F \sim \exp \left(\frac{4}{3}\right), c=1, \beta_{0}=0$. 


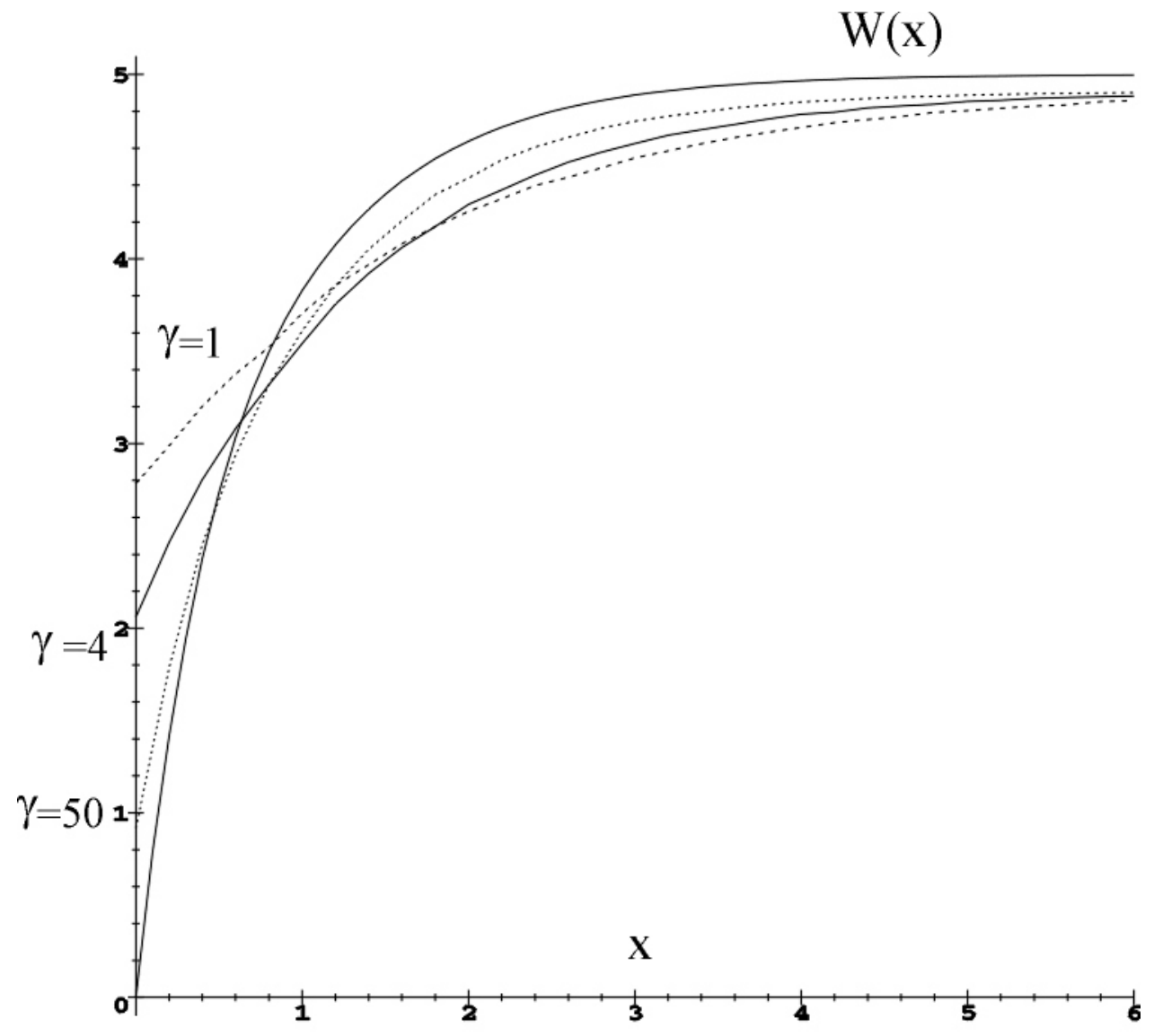

fig 3.1.: $W(x), V_{\varphi^{*}}^{\gamma}(x)$ for $\gamma=1,4,50$, $\lambda=0.5, F \sim \exp (1), c=1.5, \beta=0.1, \beta_{0}=0, \Delta=0.5$. 